\title{
Referred sensations in chronic pain patients
}

\author{
Joel Katz and Ronald Melzack
}

\begin{abstract}
Summary This clinical note describes an unusual phenomenon of referred sensation reported in a sample of 98 chronic pain patients during electrical stimulation. Thirty-nine percent reported a variety of sensations referred to different parts of the body. Of these, $74 \%$ reported the sensations referred to the painful region. Among the sensations were paresthesias, pain, temperature changes, and pressure or constriction. The patients who had referred sensations had lower ratings of depression and had undergone more surgical operations than those who did not report referred sensations. Three case reports of patients with phantom limb pain are presented to illustrate the vividness with which these sensations are experienced. These data suggest that deafferentation due to disease, injury or other lesions of the CNS lead to a hypersensitivity and an increased likelihood of referred pain of long duration.
\end{abstract}

Key words: chronic pain; referred sensations

A study of auriculotherapy showed that electrical stimulation applied to the outer ear failed to produce significant relief of chronic pain when compared to a control condition [2]. However, the patients reported an astonishingly large number of referred sensations. In order to study this phenomenon more closely, we reviewed the clinical records of the 36 patients who participated in the study on auriculotherapy as well as 62 others seen at the Pain Center of the Montreal General Hospital who had received electrical stimulation applied at the outer ears, face, and torso for the relief of pain. The patients in the auriculotherapy study received electrical stimulation for 10 min each session at $4 \mathrm{~Hz}$ using a $50 \%$ duty cycle and a mean intensity of $107 \mu \mathrm{A}$. Stimulation parameters varied across patients and sessions for the remaining 62 subjects.

The subjects of the present study were 98 patients ( 58 women and 40 men) with chronic pain syndromes of diverse etiology. Thirty-eight patients (39\%) reported sensations referred to various parts of their body during stimulation. Among the 
TABLI: 1

A COMPARISON OF PATIENTS WHO REPORTED REFIRREI SENSATIONS WITH THIOSE: WHO DID NOT

\begin{tabular}{lcc}
\hline & $\begin{array}{l}\text { Referred } \\
\text { sensations (N) }\end{array}$ & $\begin{array}{l}\text { No referred } \\
\text { sensations (N) }\end{array}$ \\
\hline Age & $55.1(38)$ & $54.6(60)$ \\
Chronicity of pain (years) & $5.6(30)$ & $7.1(60)$ \\
Number of surgeries & $3.5(35)$ & $2.7(57)$ \\
MMPI Hs & $75.0(16)$ & $75.0(25)$ \\
MMPI D & $70.0(16)$ & $81.0(25)$ \\
MMPI Hy & $71.0(16)$ & $74.0(25)$ \\
& & $\mathrm{N}$ \\
How pain began & $\mathrm{N}$ & 16 \\
Following an accident & 10 & 10 \\
Following surgery & 11 & 7 \\
Following an illness & 2 & 24 \\
Suddenly & 13 & \\
\hline
\end{tabular}

i $(90)=2.1, P<0.05$.

b MMPI scores were available for only 16 of the 38 patients that reported referred sensations and 25 of the 60 that did not.

C $t(39)=2.54, P<0.01$.

reported sensations were tingling, itching, pressure, 'glowing' and changes in temperature and pain intensity.

In order to distinguish the patients who reported referred sensations from those who did not, we compared them on a number of relevant variables shown in Table I. Patients who reported referred sensations had undergone significantly more surgical procedures and reported significantly lower levels of depression (as measured by the MMPI-D scale) than those who did not report these sensations ( $t$ $(90)=2.1, P<0.05$, and $t(39)=2.54, P<0.01$, respectively). Since all but one of the patients with phantom limb pain reported referred sensations, it was possible that the greater number of surgical procedures we observed was inflated by these patients, reflecting the fact that a necessary precondition for phantom limb pain is amputation. A comparison of the mean number of surgical procedures in amputees with that in the remainder of the group who reported referred sensations was not significant ( $t(34)=0.04, P=0.97$ ). Thus, the finding that the patients who reported referred sensations had undergone significantly more surgical procedures than those who did not report these sensations does not appear to be an artifact produced by the patients with phantom limb pain.

Fig. 1 shows the 4 diagnostic groups in which more than $30 \%$ of patients reported referred sensations. We have classified the reported sensations into 4 distinct types: (1) paresthesias (tingling, itching, 'pins and needles,' numbness, electric shock); (2) increases in pain intensity; (3) changes (increases or decreases) in the perceived temperature of some part of the body, and (4) a feeling of pressure or constriction. Fig. 2 shows the distribution of these 4 types of sensations across the total sample, 


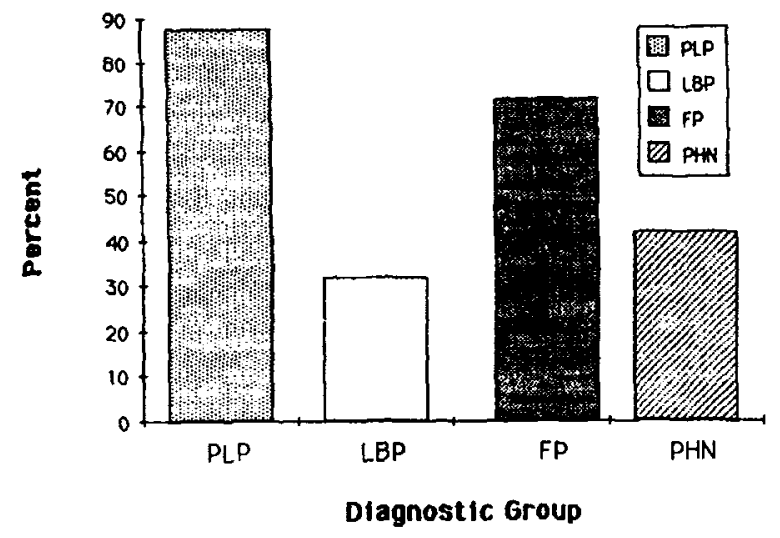

Fig. 1. Percentage of patients with phantom limb pain (PLP), low-back pain (LBP), facial pain (FP), and post-herpetic neuralgia (PHN) that reported referred sensations during electrical stimulation.

and within the 4 diagnostic groups shown in the previous figure. With the exception of the patients with low-back pain, the most common sensations experienced were paresthesias. Patients with phantom limb pain and those with low-back pain reported the most varied types of sensations whereas patients with post-herpetic neuralgia experienced only paresthesias and pain (usually described as an intensification of the burning pain they were seeking to relieve).

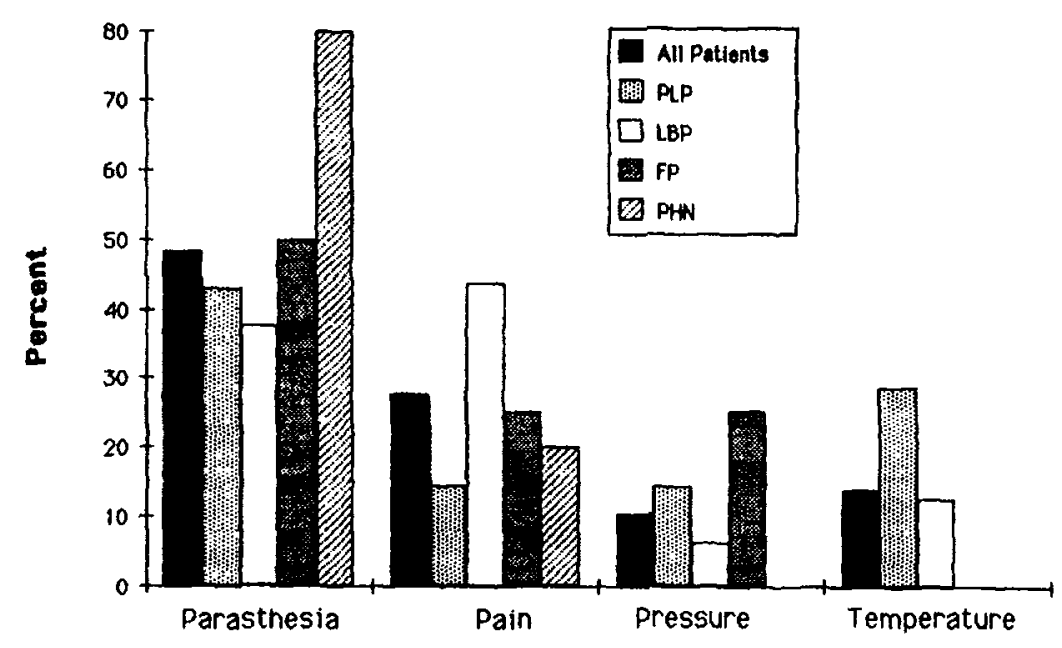

Type of Sensation

Fig. 2. Percentage of the total number of referred sensations for each diagnostic group displayed according to the type of sensation. Since many patients reported more than one type of referred sensation, the total number of responses exceeds the number of patients. PLP, phantom limb pain; LBP, low-back pain; FP, facial pain; PHN, post-herpetic neuralgia. 
Seventy-four percent of the patients who reported referred sensations experienced them in the painful region: 26\% reported that they were referred to other body parts. However, for both groups, the pattern of referral was the same: overall. the sensations were referred to the extremities, the low-back region, and the groin more frequently than to other parts of the body.

The following case descriptions illustrate the various types of referred sensations reported by 3 patients with phantom limb pain.

\section{Case 57}

This patient was a 55-year-old manual laborer who sustained a complete forequarter amputation of his right shoulder as a result of a work accident 2 years prior to his referral to the pain center. He had suffered from continuous phantom limb pain ever since the accident.

His phantom limb consisted solely of the lower arm and hand, with a gap between the stump and the beginning of the phantom elbow (Fig. 3). He described the limb as suspended in space. Whenever he walked, it behaved as a real limb would, swinging naturally back and forth in synchrony with his contralateral leg. Such automatic movements of the limb were common. He described how, while walking down a flight of stairs, he instinctively reached out to grab the bannister with his phantom arm to avoid a fall, only to find himself lying on the landing below. In contrast, it required considerable effort for him to willfully move the phantom limb: he reported that he was able to partially open the hand, but this was a slow and frustrating process that required enormous concentration.

He described the pain as a throbbing across the dorsum of the phantom hand. The thumb and index finger were especially sensitive. On his first visit to the pain center one of his primary complaints was that he had great trouble falling asleep at night, for if he lay on his right side he would experience a sharp increase in pain, and when he turned over, the phantom arm would rise upward like a helium-filled balloon until it was fully extended over his head. After several minutes in this posture, his arm would become heavy with fatigue, and an unbearable pain would ensue, forcing him to shift position once again.

On the first session, during electrical stimulation applied at a point on his left ear, he reported a warm sensation that traveled down his phantom arm and into the hand which then began to swell. Later, when the stimulus was applied to the same point on his right ear, he remarked that his phantom limb had suddenly telescoped into the stump so that the once empty space was now occupied by the lower arm. On the following day, during stimulation of the same point, he reported a further abrupt retraction of the limb, leaving the wrist and hand protruding from the stump. The patient subsequently reported an improvement in his sleep and no longer experienced involuntary tendencies to use the phantom in moments of urgency. The shortened version of the phantom was maintained at a 5-month follow-up visit, and more recently in a telephone interview 3 years later. 

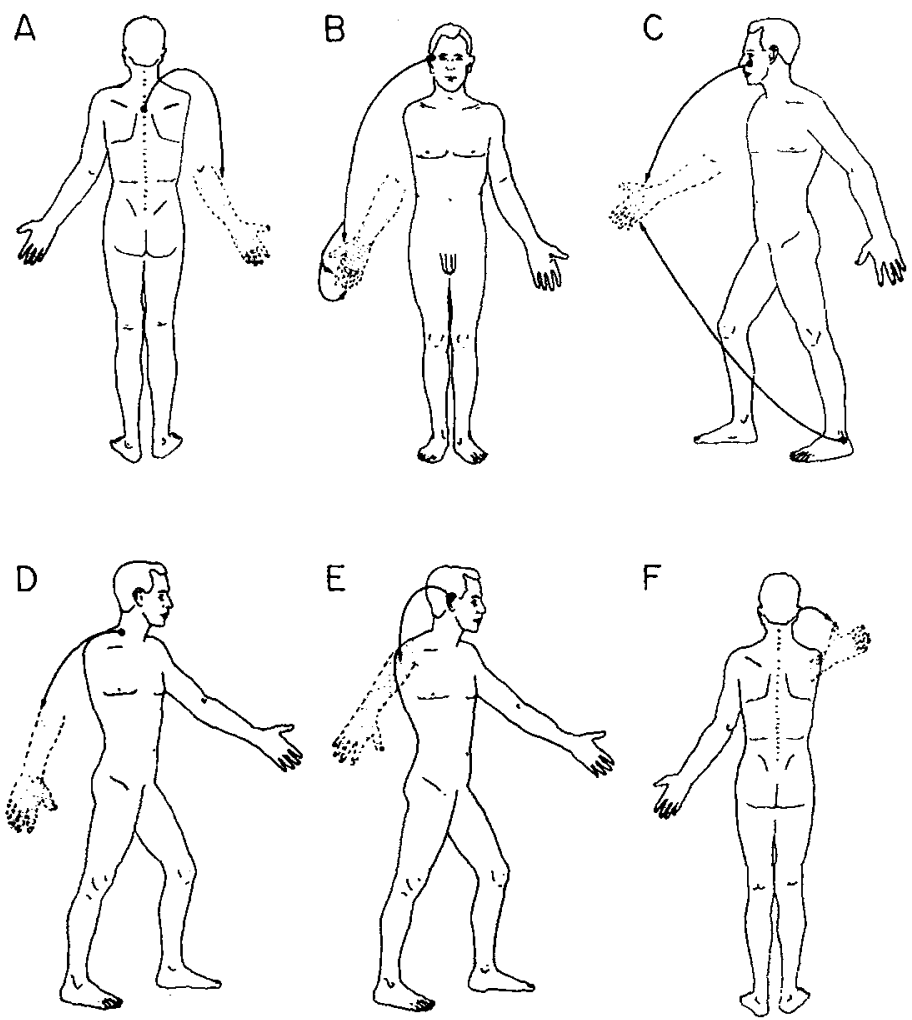

Fig. 3. Referred sensations reported by a patient (case 57) with phantom limb pain during electrical stimulation. Dots represent points on the body where stimulation was applied. Arrows indicate where the sensations were referred. A: stimulation applied at a point on the midline of the patient's upper back produced an increase in the paresthetic sensations that usually defined his phantom limb. B: during stimulation applied at a point on his right ear, the patient reported that the phantom hand became warm and the fingers began to move. $C$ : increased paresthesias in the phantom arm resulted from stimulation applied at points to either side of his nose and as far away as the left ankle. D: during stimulation to a point just above the clavicle, the patient reported that his phantom hand began to swell and become warm. E: in response to stimulation at a point on his right ear, he reported that his phantom arm had telescoped into the stump so that the elbow protruded from the stump. F: later, stimulation to the same point resulted in a further retraction of the phantom leaving only the wrist and hand protruding from the stump.

\section{Case 60}

This patient was a 60 -year-old man with bilateral lower extremity phantom pain. The pain consisted of intense, rhythmic throbbing in the big toes of both phantom feet. As the pain increased in severity, the throbbing became the focus of frequent, sharp, lightning-like bursts of pain which shot up the length of his phantom legs, causing an uncontrollable jerking of the stumps, leaving him visibly shaken. 
This patient was seen on 7 occasions. Without exception. within $3 \mathrm{~min}$ of stimulation applied at either ear, he reported that both phantom limbs would vanish. With the disappearance of his phantom legs, any pain he had previously been experiencing was also reportedly abolished. This state of 'phantom-less' limbs persisted for several hours after stimulation until he would suddenly notice that his phantom limbs had returned.

During the interval of stimulation that preceded the disappearance of his phantom legs, he remarked that he was experiencing sensations in new and unfamiliar parts of his limbs. including a tingling in both phantom soles and heels. These were parts that he had never felt after he lost his legs and were not features that ordinarily defined his phantom limbs.

\section{Case 79}

This patient was a 54-year-old woman who had been referred to the Pain Center because of severe and unrelenting phantom limb pain of 3 years' duration. In 1962 she had received an over-exposure of irradiation during a course of cobalt radiotherapy following a left radical mastectomy for breast cancer.

Over the next 8 years she developed progressive numbness, atrophy, and paresis of the left arm with marked necrosis and ulcerations of the left chest wall and arm which required repeated debriding and drainage. By 1978 there was complete numbness and paralysis of the left arm. A left fore-quarter amputation was performed, and the area of ulceration on the chest was repaired by skin grafts using tissue from the amputated arm. Immediately after the amputation she reported a distinct and severely painful phantom arm.

She described the usual position of her phantom arm as one in which the upper arm rested at her side. The elbow was bent at an angle slightly less than $90^{\circ}$ and the hand lay below her right breast.

The limb was always painful. The pain consisted of an intense, hot, generalized throbbing of the phantom arm. The fingers and hand would often swell out of proportion to the rest of the arm, producing an intense constricting pain. When the pain reached an intolerable level, she described how the entire limb would extend rigidly outward at shoulder height until the pain subsided.

She was seen on 5 occasions. On each of these, she reported a considerable increase in the intensity of phantom sensations during electrical stimulation applied at points on her ears, face, and body (Fig. 4). On the first session, when stimulation was applied to a point on her left ear, she reported feeling tingling sensations referred to parts of her phantom arm that she had never experienced before. She described fleeting, yet distinct, sensations along the anterior aspect of her elbow, and an itching of the palm and fingers.

On another occasion when stimulation was applied to the ear she reported, with some surprise, a 'pleasant sensation of draining' in her phantom arm, and remarked that it was very much like the sensation she had experienced prior to the amputation when the ulcers on her arm had been drained. 

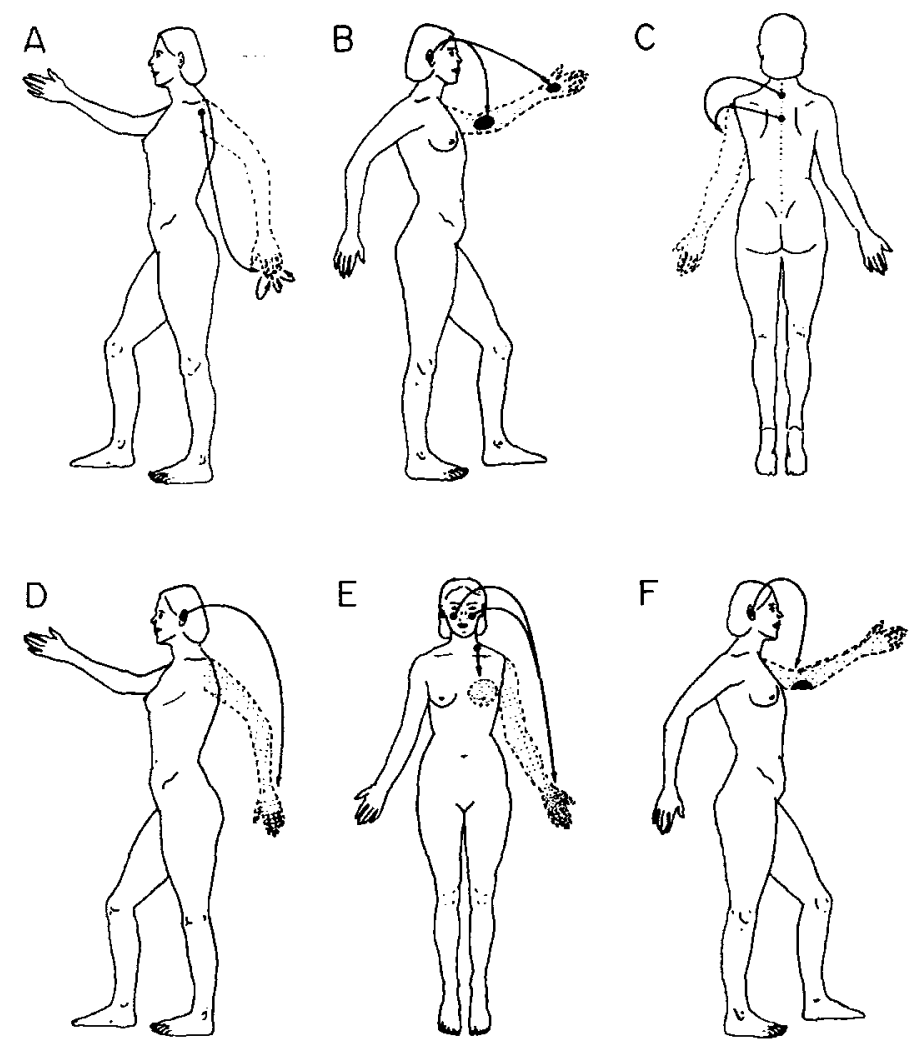

Fig. 4. Referred sensations reported by a patient (case 79) with phantom limb pain during electrical stimulation. To simplify the illustration of the referred patterns, the phantom limb is depicted as being at the side rather than bent at the elbow and across the chest as the patient actually reported. Dots and arrows represent stimulation and referral sites, respectively. A: stimulation applied at a point on the stump produced a sensation in the phantom arm that consisted of electric shock-like activity that jumped from finger to finger. B: when stimulation was applied to her right ear, she remarked that the sensations were referred to the inside of her phantom elbow and parts of the palm, neither of which she had experienced before. $\mathrm{C}$ : an increase in the paresthetic sensations in her phantom arm resulted from stimulation to both points at the midline on her upper back. D: stimulation of a point on her left ear produced a sensation of heat 'rushing' into the phantom hand. E: stimulation applied on either side of the nose produced a cool sensation in the phantom hand; when applied to a point just above the clavicle on the left side she reported a distinct sensation of a phantom breast. F: during stimulation applied to a point on the right ear the patient reported that her phantom elbow became warm and she described a pulsing sensation that traveled down the wrist and into the thumb.

Stimulation applied just above the left clavicle produced a distinct awareness of a phantom breast, another sensation that she had not experienced before. Applying the stimulus approximately $1.5 \mathrm{~cm}$ to the left and right of her nose produced a rushing sensation of 'coolness' in the phantom hand and a tingling in the palm. On the third session, she described a pulsing sensation that traveled down the wrist and into the phantom thumb. Later, stimulation applied to a point on the stump produced a spread of activity which she described as an 'electric shock-like' 
sensation that jumped from one digit to the next. beginning at the index finger. and continuing until the entire hand was tingling. A telephone follow-up 3 years later indicated that she continued to apply electrical stimulation to the outer ears for 20 min each day with satisfactory pain relief for up to $3 \mathrm{~h}$.

\section{Discussion}

These findings suggest that there is a convergence and summation of somatosensory inputs from the outer ear, face and torso at excitatory neuron pools in the central nervous system that underlie the referred sensations these patients experience and give rise to multiple sensations that are perceived to originate in the periphery. This is most vividly demonstrated by the reports of the 3 patients with phantom limb pain. Not only did they all report an overall increase in the kind and vividness of phantom sensations during stimulation, but two of them experienced parts of their phantom limbs for the first time. The most common sensation reported by patients who receive electrical stimulation of the thalamus and the medial lemniscal pathway is one of paresthesias whereas electrical stimulation of the spinothalamic pathway invariably produces referred sensations of temperature change, and only rarely, pain [5]. However, in patients who suffer from chronic pain, electrical brain stimulation at almost any site reproduces their pain. This state of CNS hypersensitivity seems to characterize patients with deafferentation types of pain, and these make up the majority of the present sample.

A 'central' explanation of these referred sensations is also consistent with the remarkable sensory memory re-experienced by case 79 who reported feeling 'a pleasant sensation of draining' in her phantom limb that she had originally experienced years earlier prior to the amputation of her arm. Nathan [3] described a similar finding after applying noxious stimuli to the stump of an amputee, who later re-experienced the pain of a skating injury that he had sustained 5 years earlier when his leg was intact. More recently, Jensen et al. [1] observed that as long as 2 years after the amputation, $10 \%$ of their sample of amputees described the localization and character of their phantom limb pain as similar to the pain they experienced before the amputation. These findings indicate that sensory memory traces are laid down at the time of injury, and that under the appropriate conditions they may be re-activated and the original sensations re-lived.

It is also possible that the reports of referred sensations during electrical stimulation reflect a central mechanism that acts on peripheral structures. The changes in temperature sensation referred to various parts of the body suggest that stimulation may result in reduced sympathetic nervous system activity and a consequent increase in peripheral blood flow. Reports of pain relief following stimulation in patients with phantom limb pain (e.g., cases 60 and 79) may be related to post-stimulation changes in autonomic activity or they may be 'placebo'induced [2]. Alternatively, they may reflect a contrast effect in which the heightened activity level of phantom limb sensations brought about by stimulation is reduced following stimulation. The perceived reduction in the level of phantom limb activity 
may be interpreted as pain relief. Further research is underway to assess these possibilities by measuring pain levels and autonomic activity during electrical stimulation.

Patients who reported referred sensations were less depressed and had undergone more surgical procedures than those who did not report these sensations. Given the retrospective nature of this study it is not possible to determine the temporal onset of these variables in relation to the referred sensations. One possibility is that the patients who report these sensations, in general, are more vocal, tend to report their internal states more frequently, and as a result end up receiving surgery more often. Given their tendency to be more vocal, one might also expect them to experience lower levels of depression. On the other hand, it is equally likely that repeated surgical interventions create an abnormal central state in which referred sensations arise. Nathan [4] reported that shortly after a cordotomy, 18\% of his patients described a reference of sensation in which a noxious stimulus applied within the analgesic region was felt as pain at the mirror image point on the other side of the body.

These data, taken together, suggest that deafferentation due to disease, injury or other lesions of the CNS leads to a hypersensitivity of the CNS and an increased probability of referred pain of long duration.

\section{Acknowledgements}

This study was supported by Grant A7891 from the Natural Sciences and Engineering Research Council of Canada, and by a Medical Research Council of Canada Studentship awarded to the first author.

\section{References}

1 Jensen, T.S., Krebs, B., Nielson, J. and Rasmussen, P., Immediate and long-term phantom limb pain in amputees: incidence, clinical characteristics and relationship to pre-amputation pain, Pain, 21 (1985) 267-278.

2 Melzack, R. and Katz, J., Auriculotherapy fails to relieve chronic pain: a controlled crossover study, J. Amer. med. Ass., 251 (1984) 1041-1043.

3 Nathan, P.W., Pain traces left in the central nervous system. In: C.A. Keele and R. Smith (Eds.), The Assessment of Pain in Man and Animals, Livingstone, London, 1962, pp. 129-134.

4 Nathan, P.W., Reference of sensation at the spinal level, J. Neurol. Neurosurg. Psychiat., 19 (1956) 88-100.

5 Tasker, R.R., Organ, L.W. and Hawrylyshyn, P.A., The Thalamus and Midbrain of Man, Thomas, Springfield, IL, 1982, 505 pp. 